\title{
Polarization-correlated photons from a positively charged quantum dot
}

\author{
Y. Cao, ${ }^{1,2}$ A. J. Bennett, ${ }^{1}$ I. Farrer, ${ }^{3}$ D. A. Ritchie, ${ }^{3}$ and A. J. Shields ${ }^{1}$ \\ ${ }^{1}$ Toshiba Research Europe Limited, Cambridge Research Laboratory, 208 Science Park, Milton Road, Cambridge CB4 OGZ, United Kingdom \\ ${ }^{2}$ Department of Physics, Imperial College London, Prince Consort Road, London SW7 2AZ, United Kingdom \\ ${ }^{3}$ Cavendish Laboratory, Cambridge University, J. J. Thomson Avenue, Cambridge CB3 OHE, United Kingdom
}

(Received 13 May 2015; revised manuscript received 14 July 2015; published 7 August 2015)

\begin{abstract}
Polarized cross-correlation spectroscopy on a quantum dot charged with a single hole shows the sequential emission of photons with common circular polarization. This effect is visible without magnetic field, but becomes more pronounced as the field along the quantization axis is increased. We interpret the data in terms of electron dephasing in the $X^{+}$state caused by the Overhauser field of nuclei in the dot. We predict the correlation time scale can be increased by accelerating the emission rate with cavity QED.
\end{abstract}

DOI: 10.1103/PhysRevB.92.081302

PACS number(s): 78.66.Fd, 78.67.Hc

Spins in quantum dots (QDs) provide a promising platform for manipulating and storing quantum information in the solid state. Optical measurements have demonstrated spin preparation [1,2] coherent spin control [3], and electronspin-photon entanglement [4,5]. There are also proposals for achieving photon entanglement [6] and nondestructive measurement of photons [7] using charged QDs. However, the time evolution of the carrier spin is unavoidably affected by the $10^{4}-10^{5}$ nuclei in the dot, all with nonzero spin. One example of the utility of the electron-nuclear interaction is its use in spin pumping the hole into the spin down state in zero external field, by pumping on the spin up state of $X^{+}$[2]. From a fundamental point of view then, the hyperfine interaction provides an interesting system for manipulating a mesoscopic nuclear ensemble and observing its dynamics.

It has now been established that the electron-nuclear hyperfine interaction is dominated by the contact interaction and is isotropic in a QD [8]. The dynamics of this interaction manifests itself in studies of polarized photoluminescence $[9,10]$. Contrastingly, the hole's $p$-like wave function has a node at each nucleus, leaving the dipole-dipole interaction between the hole and nuclear spins to dominate [11]. This interaction has a strength one order of magnitude below that of the electron $[12,13]$. Thus, there has been interest in using the hole spin as a quantum bit with reduced decoherence. Direct measurements of the hole-spin relaxation time in a vertical magnetic field $T_{1}^{h}$ have shown it is hundreds of microseconds [2,14]. Without applied magnetic field, some experiments suggest the hole spin $T_{1}^{h}$ time is $13 \mathrm{~ns}$ [8]. Several studies have now estimated the hole dephasing time $T_{2}^{h}$ in magnetic field is approximately $1 \mu \mathrm{s}[3,15]$.

We study here the emission from the $X^{+}$state in a dot deterministically charged with a hole using a diode [Fig. 1(a)]. We show photons from this transition display polarization correlation over a time scale one order of magnitude greater than the radiative lifetime when excited by a linearly polarized laser. This time is short relative to some reports of the hole-spin polarization lifetime [14], and we show that this is a result of dephasing caused by the electron when the system is excited. We investigate the magnitude of the effect as a function of applied external magnetic field and radiative lifetime.
The $X^{+}$consists of two holes in a singlet $S=0$ state and an electron. The zero net-hole spin ensures the electron-hole anisotropic exchange interaction is absent. In zero magnetic field, the $X^{+}$eigenstates are degenerate and labeled by the spin of the electron [Fig. 1(b)] [16,17]. Radiative decay from $X^{+}$ to the single hole ground state occurs with a change in total angular momentum \pm 1 , the polarization of the photon being correlated with the initial and final spin state. In a dot where the hole is "heavy" ( $\left.m_{j}= \pm 3 / 2\right)$ only the vertical transitions in Fig. 1(b) are allowed: The detection of a left-handed photon $(L)$ photon ensures the decay occurred by the left-hand transition on Fig. 1(b).

The strain, shape anisotropy, and inversion asymmetry in InGaAs/GaAs QDs ensures the optically active transition has a mixed heavy-hole $\left(m_{j}= \pm 3 / 2\right)$ and light-hole $\left(m_{j}= \pm 1 / 2\right)$ character [18,19]. The state is given by $\phi_{ \pm}=(| \pm 3 / 2\rangle+\beta \mid \mp$ $1 / 2\rangle) / \sqrt{\left(1+\beta^{2}\right)}$, which we denote $\Uparrow$ and $\Downarrow$. Recombination of an electron and a mixed heavy/light hole now results in elliptically polarized photons from $X^{+}$, and $\beta$ may be determined from the emission pattern [Fig. 1(d)] [18]. Within the sample studied, $\beta$ values from 0.02 to 0.20 are typical, and for the data shown here, $\beta=0.092$ [Fig. 1(d)].

The diode for controlled charging has a $20 \mathrm{~nm} \mathrm{GaAs}$ tunnel barrier between the dot and $p$ contact [Fig. 1(a)]. A 75\% AlGaAs barrier on the $n$ side prevents electron charging, so the $X^{+}$dominates at 1.2-1.3 V. Emission from $X^{+}$at an energy of $1349.2 \mathrm{meV}$ is excited quasiresonantly by a linearly polarized laser at $1317.2 \mathrm{meV}$. This excitation scheme equally excites both transitions in Fig. 1(b) and the absence of spin pumping ensures there is no buildup of nuclear spin polarization, but it does not populate other carrier combinations such as neutral and negatively charged excitons [Fig. 1(c)].

The experiment is shown in Fig. 2(a). After filtering, the emission is passed to polarization-maintaining fiber optics which enable four simultaneous measurements of correlation in a basis selected by the quarter-wave plate (QWP) and the polarizing coupler (PBS).

Figure 2(b) presents correlations recorded at zero external field at an excitation power $\times 10$ below saturation, in the circular basis. Comparing the sum of the two copolarized and cross-polarized measurements $\left[g_{\mathrm{co}}(t)\right.$ in black and $g_{\text {cross }}(t)$ in red, respectively], we see a clear difference. Note that both $g_{\text {co }}(t)$ and $g_{\text {cross }}(t)$ show a reduced signal within $\sim 1$ ns of 
(a)

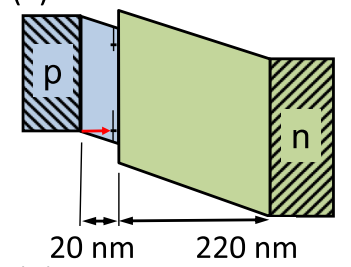

(b)

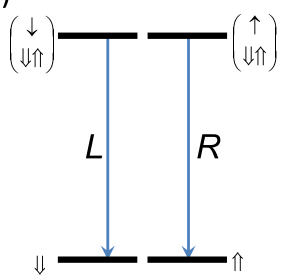

(c)

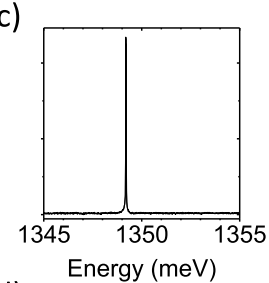

(d)

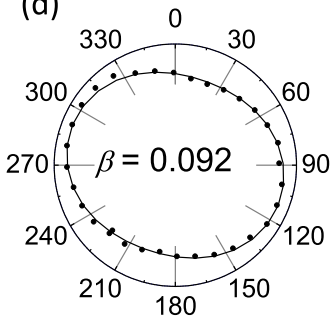

FIG. 1. (Color online) (a) Schematic band structure of the holecharging diode when the dot contains a single hole. (b) Energy level diagram for the $X^{+}$at zero magnetic field. (c) Spectrum under quasiresonant excitation at $1.2 \mathrm{~V}$. (d) Emission pattern for an $X^{+}$ transition showing $\beta=0.092$.

zero-time delay due to the antibunched nature of the light. Outside the central $\sim 1$ ns there is an enhanced probability of the source emitting two photons of the same circular polarization over the case of emitting photons of opposite circular polarization.

The degree of polarization correlation $C(t)$ is defined as $C(t)=\left[g_{\text {co }}(t)-g_{\text {cross }}(t)\right] /\left[g_{\text {co }}(t)+g_{\text {cross }}(t)\right]$ from which a least squares fit with a function $C(t)=C_{0} \exp \left(-|t| / \tau_{d}\right)$
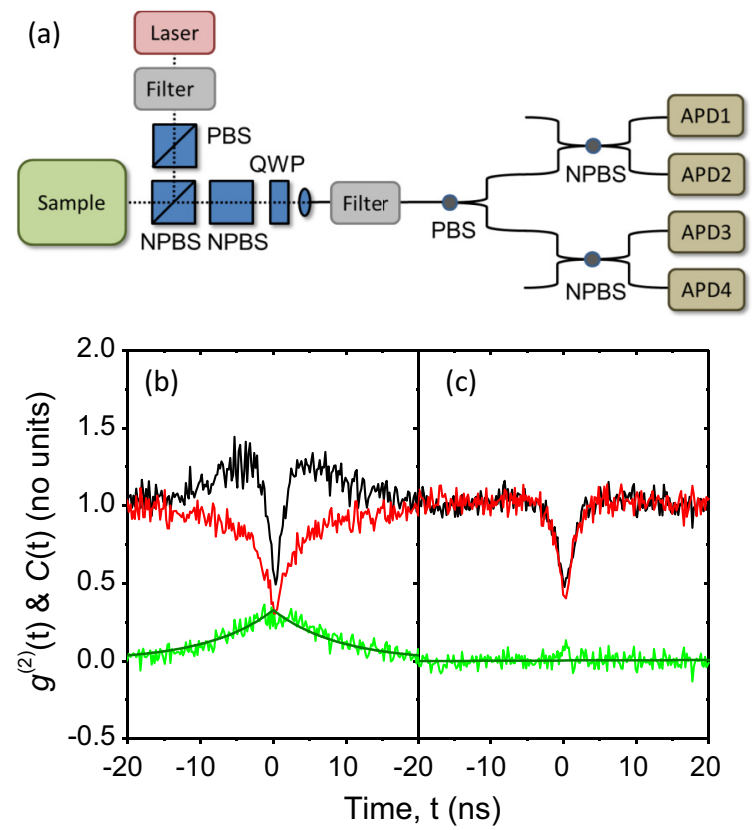

FIG. 2. (Color online) (a) Apparatus to measure the polarization correlation from a dot. (b) Circular copolarized and cross-polarized emission correlation from $X^{+}$at zero external field, $g_{\text {co }}(t)$ (black) and $g_{\text {cross }}(t)$ (red), respectively. Extracted degree of polarization correlation $C(t)$ (green). (c) The same measurement made in the linear detection basis.

extracts the polarization correlation at zero delay $C_{0}$ and the time scale $\tau_{d}$. Empirically, this function is a good fit to $C(t)$ [Fig. 2(b)]. $C_{0}=0.33 \pm 0.01$ and the decay time of the correlation $\tau_{d}=9.0 \pm 0.4 \mathrm{~ns}$. In contrast, measurements in the linear-polarization basis $(H / V)$ show an absence of polarization correlation [Fig. 2(c)].

Nonzero heavy-light-hole mixing is an obvious source of reduced polarization correlation. Taking the heavy:light-hole oscillator strength of $3: 1[18,19]$, we see that recombination of a $\phi_{+}$hole and an electron in the $X^{+}$level leads to an elliptical photon with state $\alpha \sqrt{3}|L\rangle+\beta|R\rangle$, and a $\phi_{-}$hole. Conversely, decay involving a $\phi_{-}$hole and an electron leads to a $\propto \sqrt{3}|R\rangle+\beta|L\rangle$ photon. The measurement in Fig. 2(b) is in the circular basis, so detection of a left-handed photon implies the decay came from $\phi_{+}$with $3 /\left(3+\beta^{2}\right)$ probability. In the absence of dephasing in the upper or lower states, this reduces the probability of obtaining sequential left-left photodetections to $\left(3^{2}+\beta^{4}\right) /\left(3+\beta^{2}\right)^{2}$. For this QD, $\beta=$ 0.092 , so the probability of copolarized photon emission is reduced to 0.994 . This is higher than we have measured, so we conclude an additional factor must be included.

In fact, the data can be explained by the fast dephasing of the electron spin in the upper state, which dominates any dephasing from the hole spin. A coincidence detection event arises as follows: The transition emits a photon that is detected with circular polarization and the hole spin is left in the corresponding state. Some time later, the system is reexcited to the upper state, where electron spin dephasing occurs during the radiative lifetime of the $X^{+}$state, following which a second photon is emitted from the spontaneous decay. These two photons form a single coincidence in Fig. 2(b). We stress that our model implicitly assumes the hole-spin lifetime is greater than the measured $\tau_{d}$, though we envisage that in future experiments that reduce the effect of electron dephasing, it will be necessary to include the contribution of the hole.

Our studies provide four pieces of evidence that electronspin dephasing is the factor limiting the polarization correlation. First, the degree of polarization correlation from the $X^{+}$observed in Fig. 2(b) is $1 / 3$. When excited, the unpaired electron spin evolves through a hyperfine interaction with the nuclei. Only those nuclear field fluctuations in the two directions perpendicular to the spin will cause precession. If this precession is faster than the radiative lifetime of the upper state, its effect is to randomize the spin. The electron spin parallel to the nuclear field is preserved. Thus, the mean spin projection along $\mathbf{z}$ is reduced to $1 / 3$. Second, the time scale over which polarization correlation is observed is inversely proportional to the pump rate, as shown in Fig. 3. This cannot be explained by dephasing occurring in the ground state. The increased excitation increases the number of times the system is excited between photon detection events, and this increases the rate at which the polarization correlation is lost. Third, there is no polarization correlation in the linear basis [Fig. 2(c)]. This is consistent with a dephasing of the electron-spin state in a time faster than the $X^{+}$radiative lifetime. Finally, we shall show that the change in $C_{0}$ with magnetic field is explained by the dynamics of the electron spin.

We next discuss the application of a Faraday magnetic field, which removes the degeneracy of the upper and lower states, 


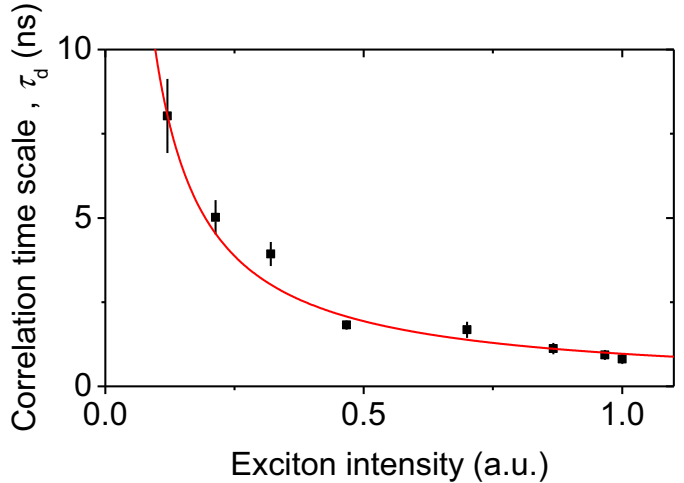

FIG. 3. (Color online) A measurement of the time scale of correlation at zero external magnetic field $\tau_{d}$ as a function of the normalized intensity of the source. The data are fitted with an inverse relationship.

shown in Fig. 4(a). The net field experienced by the spins is the sum of the external field $B_{\text {ext }}$ and the internal nuclear field $B_{N}$. This stabilizes the electron spin along $\mathbf{z}$ and causes it to precess about the sum of the two fields, which is predominantly along the $\mathbf{z}$ when the $\left|B_{\text {ext }}\right|>B_{N}$. Thus, the application of vertical field increases the value of $C_{0}$, as shown in Fig. 4(b). Figure 4(c) plots the polarization-correlation time scale $\tau_{d}$ versus magnetic field at constant laser intensity. This value changes from $9.0 \pm 0.4 \mathrm{~ns}$ at zero field to $14.5 \pm 0.5 \mathrm{~ns}$ at $300 \mathrm{mT}$.

A model of the dephasing of electron spin in QDs was presented by Merkolov, Efros, and Rosen [20]. In this framework it is assumed that on time scales below $1 \mu \mathrm{s}$ the hyperfine interaction between the electron spin and the nuclei in the dot can be considered semiclassically as a "frozen" magnetic field, of finite variance, but no directional preference. The electron $g$ factor is assumed isotropic. The time evolution of the electron spin $\mathbf{S}(t)$ (initially along $\mathbf{S}_{0}$ ) is
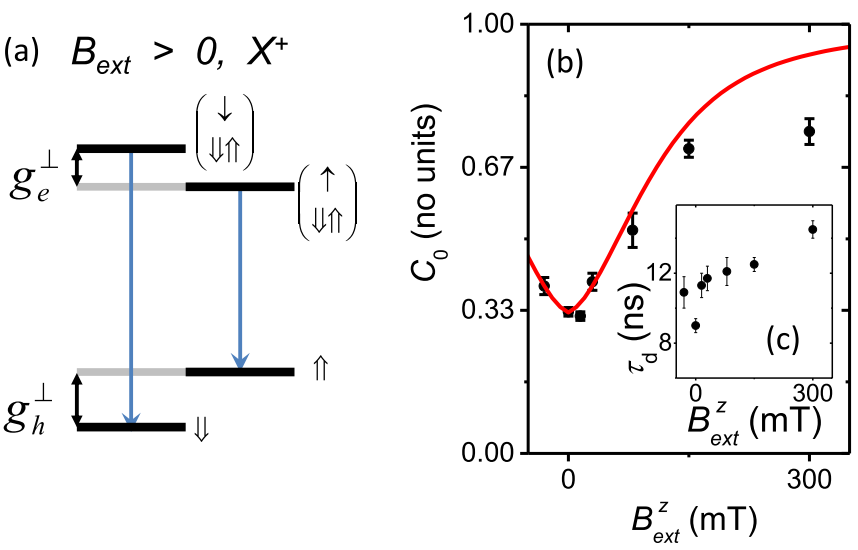

FIG. 4. (Color online) Energy level diagram for the $X^{+}$transition with finite $z$ magnetic field. (b) The degree of correlation $C_{0}$ as a function of external Faraday magnetic field (black points). The calculated variation with field is shown as a red line. (c) Variation in the time scale of correlation $\tau_{d}$. (a)

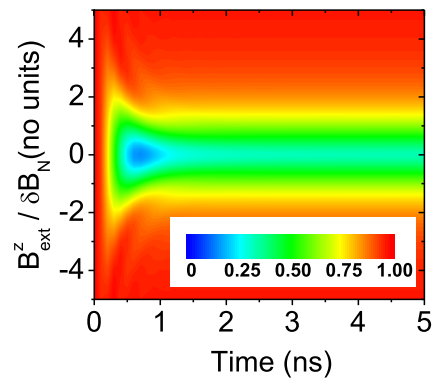

(b)

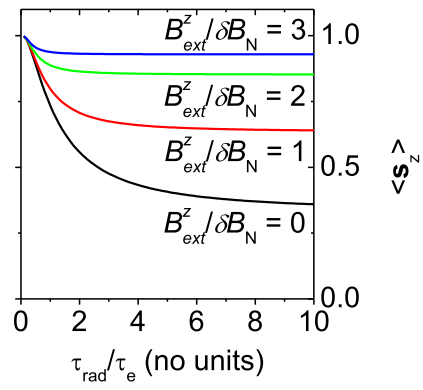

FIG. 5. (Color online) (a) Projection of the electron spin along the $z$ direction as a function of magnetic field and time, assuming a nuclear field fluctuation of width $\delta B_{N}=100 \mathrm{mT}$ and an electron $g$ factor of 0.5 [21]. (b) The averaged projection of the $z$ component of the spin over the radiative lifetime, scaled in terms of the electron-spin dephasing time.

given by

$$
\begin{aligned}
\mathbf{S}(t)= & (\mathbf{S} \cdot \mathbf{n}) \mathbf{n}+\left\{\mathbf{S}_{0}-\left(\mathbf{S}_{0} \cdot \mathbf{n}\right) \mathbf{n}\right\} \cos (\omega t) \\
& +\left[\left\{\mathbf{S}_{0}-\left(\mathbf{S}_{0} \cdot \mathbf{n}\right) \mathbf{n}\right\} \times \mathbf{n}\right] \sin (\omega t), \\
W\left(B_{N}\right) \propto & \exp \left[-\frac{\left(B_{N}\right)^{2}}{\delta B_{N}^{2}}\right],
\end{aligned}
$$

where the distribution of nuclear field strengths $W\left(B_{N}\right)$ is parametrized by the Gaussian width of fluctuations $\delta B_{N}$. Figure 5(a) shows how the spin projection along the $\mathbf{z}$ direction $S_{z}$ varies with the external magnetic field $B_{\mathrm{ext}}^{z}$ [20]. At fields of a few times $\delta B_{N}$, the spin projection along $\mathbf{z}$ is stabilized. This has not eliminated the nuclear spin fluctuations, but it has merely overwhelmed them at the cost of increased rate of precession about the net field. Any measurement along an orthogonal polarization direction will reveal the increased precession rate.

From Eq. 1(a) we extract the expected final electron-spin projection along $\mathbf{z}, S_{z}$, which is equal to the polarization correlation $C_{0}$ [solid line in Fig. 4(b)]. The only fitting parameter is the width of the fluctuations in the nuclear field $\delta B_{N}$, set to $100 \mathrm{mT}$. The dephasing time for this electron [8] is therefore $T_{\triangle}=\hbar / g_{e} \mu_{B} \delta B_{N} \sim 200 \mathrm{ps}$, which is, as expected, much less than the $1 \mathrm{~ns}$ radiative lifetime of the upper state. This provides a good fit to the data, reproducing the value of $C_{0}$ and width around zero field. The model fits less well at the higher fields. Partly, this can be explained by non zero $\beta$, but the discrepancy requires further investigation.

The extracted $\delta B_{N}$ is within the range derived from a spin-noise measurement [22] but is greater than inferred from dephasing of the $X_{0}$ state in similar dots [23]. We attribute this to the smaller wave-function extent of the electron when the dot additionally contains two holes. As $\delta B_{N}$ scales with $1 / \sqrt{(V)}$, where $V$ is the volume of spins overlapping with the wave function, there is a variation in the effective $\delta B_{N}$ between states. This is the same reason the electron $g$ factor changes in the presence of additional holes [21].

To increase $C_{0}$ one could employ a host semiconductor without nuclear spin, a QD of greater volume, or reduce the fluctuations in the nuclear field. Alternatively, 
Fig. 5(b) shows that reducing $\tau_{\text {rad }}$ to the electron-spin lifetime leads to a significant increase in polarization correlation. This could be achieved by placing the dot into a cavity that equally enhances the radiative decay, independent of polarization.

In conclusion, despite the long hole-spin coherence time in quantum dots, the emission of polarization-correlated photons from the $X^{+}$state is limited by electron-spin dephasing. A significant increase in the polarization-correlation time should be achieved by reducing the radiative lifetime of the $X^{+}$state. Additionally, the degree of polarization correlation can be increased by applying an external magnetic field greater than the nuclear field.

We thank the EPSRC for funding the molecular beam epitaxy (MBE) machine and the Controlled Quantum Dynamics Centre for Doctoral Training (CQD-CDT) which supported Y. Cao.
[1] M. Atature, J. Dreiser, A. Badolato, A. Högele, K. Karrai, and A. Imamoglu, Science 312, 551 (2006).

[2] B. D. Gerardot, D. Brunner, P. A. Dalgarno, P. Öhberg, S. Seidl, M. Kroner, K. Karrai, N. G. Stoltz, P. M. Petroff, and R. J. Warburton, Nature (London) 451, 441 (2007).

[3] K. De Greve, P. L. McMahon, D. Press, T. D. Ladd, D. Bisping, C. Schneider, M. Kamp, L. Worschech, S. Höfling, A. Forchel, and Y. Yamamoto, Nat. Phys. 7, 872 (2011).

[4] K. De Greve, L. Yu, P. L. McMahon, J. S. Pelc, C. M. Natarajan, N. Y. Kim, E. Abe, S. Maier, C. Schneider, M. Kamp, S. Höfling, R. H. Hadfield, A. Forchel, M. M. Fejer, and Y. Yamamoto, Nature (London) 491, 421 (2012).

[5] W. B. Gao, P. Fallahi, E. Togan, J. Miguel-Sánchez, and A. Imamoglu, Nat. Phys. 491, 426 (2012).

[6] C. Y. Hu, W. J. Munro, and J. G. Rarity, Phys. Rev. B 78, 125318 (2008).

[7] D. Witthaut, M. D. Lukin, and A. S. Sørensen, Europhys. Lett. 97, 50007 (2012).

[8] B. Urbaszek, X. Marie, T. Amand, O. Krebs, P. Voisin, P. Maletinsky, A. Högele, and A. Imamoglu, Rev. Mod. Phys. 85, 79 (2011).

[9] S. Cortez, O. Krebs, S. Laurent, M. Senes, X. Marie, P. Voisin, R. Ferreira, G. Bastard, J-M. Gérard, and T. Amand, Phys. Rev. Lett. 89, 207401 (2002).

[10] S. Laurent, M. Senes, O. Krebs, V. K. Kalevich, B. Urbaszek, X. Marie, T. Amand, and P. Voisin, Phys. Rev. B 73, 235302 (2006).

[11] C. Testelin, F. Bernardot, B. Eble, and M. Chamarro, Phys. Rev. B 79, 195440 (2009).

[12] E. A. Chekhovich, A. B. Krysa, M. S. Skolnick, and A. I. Tartakovskii, Phys. Rev. Lett. 106, 027402 (2011).
[13] P. Fallahi, S. T. Yilmaz, and A. Imamoğlu, Phys. Rev. Lett. 105, 257402 (2010).

[14] D. Heiss, S. Schaeck, H. Huebl, M. Bichler, G. Abstreiter, J. J. Finley, D. V. Bulaev, and D. Loss, Phys. Rev. B 76, 241306(R) (2007).

[15] D. Brunner, B. D. Gerardot, P. A. Dalgarno, G. Wüst, K. Karrai, N. G. Stoltz, P. M. Petroff, and R. J. Warburton, Science 325, 70 (2009).

[16] M. Bayer, A. Kuther, A. Forchel, A. Gorbunov, V. B. Timofeev, F. Schäfer, J. P. Reithmaier, T. L. Reinecke, and S. N. Walck, Phys. Rev. Lett. 82, 1748 (1999).

[17] M. Bayer, G. Ortner, O. Stern, A. Kuther, A. A. Gorbunov, A. Forchel, P. Hawrylak, S. Fafard, K. Hinzer, T. L. Reinecke, S. N. Walck, J. P. Reithmaier, F. Klopf, and F. Schäfer, Phys. Rev. B 65, 195315 (2002).

[18] A. V. Koudinov, I. A. Akimov, Yu. G. Kusrayev, and F. Henneberger, Phys. Rev. B 70, 241305(R) (2004).

[19] T. Belhadj, T. Amand, A. Kunold, C. Simon, T. Kuroda, M. Abbarchi, T. Mano, K. Sakoda, S. Kunz, X. Marie, and B. Urbaszek, Appl. Phys. Lett. 97, 051111 (2010).

[20] I. A. Merkulov, Al. L. Efros, and M. Rosen, Phys. Rev. B 65, 205309 (2002).

[21] A. J. Bennett, M. A. Pooley, Y. Cao, N. Sköld, I. Farrer D. A. Ritchie, and A. J. Shields, Nat. Commun. 4, 1522 (2013).

[22] A. V. Kuhlmann, J. Houel, A. Ludwig, L. Greuter, D. Reuter, A. D. Wieck, M. Poggio, and R. J. Warburton, Nat. Phys. 9, 570 (2013).

[23] A. J. Bennett, M. A. Pooley, R. M. Stevenson, I. Farrer, D. A. Ritchie, and A. J. Shields, Phys. Rev. B 84, 195401 (2011). 